\title{
High Resolution Remote Sensing Image Classification based on SVM and FCM
}

\author{
Qin $\mathrm{LI}^{\mathrm{a}}$, Wenxing $\mathrm{BAO}^{\mathrm{b}}$, Xing $\mathrm{LI}^{\mathrm{c}}$, Bin $\mathrm{LI}^{\mathrm{d}}$ \\ Department of Computer Science and Engineering, Beifang University for Nationalities, Yinchuan, \\ 750021, China \\ aemail: 825773926@qq.com, bemail: bwx71@163.com, cemail: 1142332365@qq.com, \\ demail:274022488@qq.com
}

Keywords: ALOS image; SVM; FCM; textural features

\begin{abstract}
This paper proposes a remote sensing image classification method based on multi-feature combination of the support vector machine (SVM) according to the classification problems of the high resolution remote sensing image. ALOS image is operated at two stages by this method. The first stage is to coarsely classify with fuzzy c-means (FCM) algorithm and k-means algorithm, and the second stage is to extract the textural features of the image with gray-level co-occurrence matrix (GLCM). The relevancy is selected to participate in the classification of the SVM. Experiments prove that the method is an effective and feasible remote sensing image classification method.
\end{abstract}

\section{Introduction}

How to carry out automatic classification and information extraction on the satellite remote sensing data is always an important research direction of the remote sensing application. The current classification method is mostly based on a pattern recognition technology; and the common methods mainly include the maximum likelihood algorithm, artificial neural network algorithm, clustering algorithm and the like. However, these algorithms have great limitations, namely, the ideal classification result can be obtained only when the samples tend to be infinitely great and be normal distribution. But in the actual application, the number of samples is often limited, which limits that the method cannot get ideal classification result sometimes [1].

SVM was firstly proposed by Corinna Cortes and his cooperators in 1995 [2], and it shows many unique advantages in solving small samples, nonlinear and high dimensional pattern recognitions. It can be promoted and applied to the function fitting and learning problems of other machines. Chen Liang, et al. could describe the textural features of the ground object better with multiscale texture so as to help improve classification accuracy [3]; Qian Yin, et al. proposed that the feature vector was formed by combining the spectrum and textural features, and the image was classified by machine learning [4]. However, SVM has a certain difficulty when obtaining the training samples. The artificial extraction is needed, and a lot of time is spent. Some scholars used FCM to make preliminary classification. Lu Liu, et al. proposed a method of combining FCM and FSVM. This method refer to firstly divide the feature image into several blocks, then it carried out the feature vector classification on one block containing the textural features with the FCM and carried out FSVM training on the feature vector and classification result of these blocks [5]. Guangmin Wu, et al. solved the problem that the training samples shall be selected manually by combining the advantages of FCM clustering algorithm and SVM. According to the method, FCM was firstly used to cluster, and some mixed pixels were used as the marked samples to participate in the SVM classification according to the size of each clustering region and membership matrix [6].

The classification carries out by using SVM depends on features extraction to a large extent. The remote sensing image has many features, such as spectral feature, edge feature, textural feature and shape feature, etc., and the information included in the remote sensing image is abundant. The traditional method based on the classification of the spectral feature has two phenomena, i.e. different objects with same spectrum, and same spectrum with different objects, which have a certain impact on the classification accuracy. Therefore, in this paper the original remote sensing 
image is firstly classifies with FCM, and then it is classified by combining the textural features. The method describes the contents of the remote sensing image from different aspects in more detail so as to reduce the manual intervention to realize better classification result. The specific flow of the image classification method in this paper shall be shown in Fig.1.

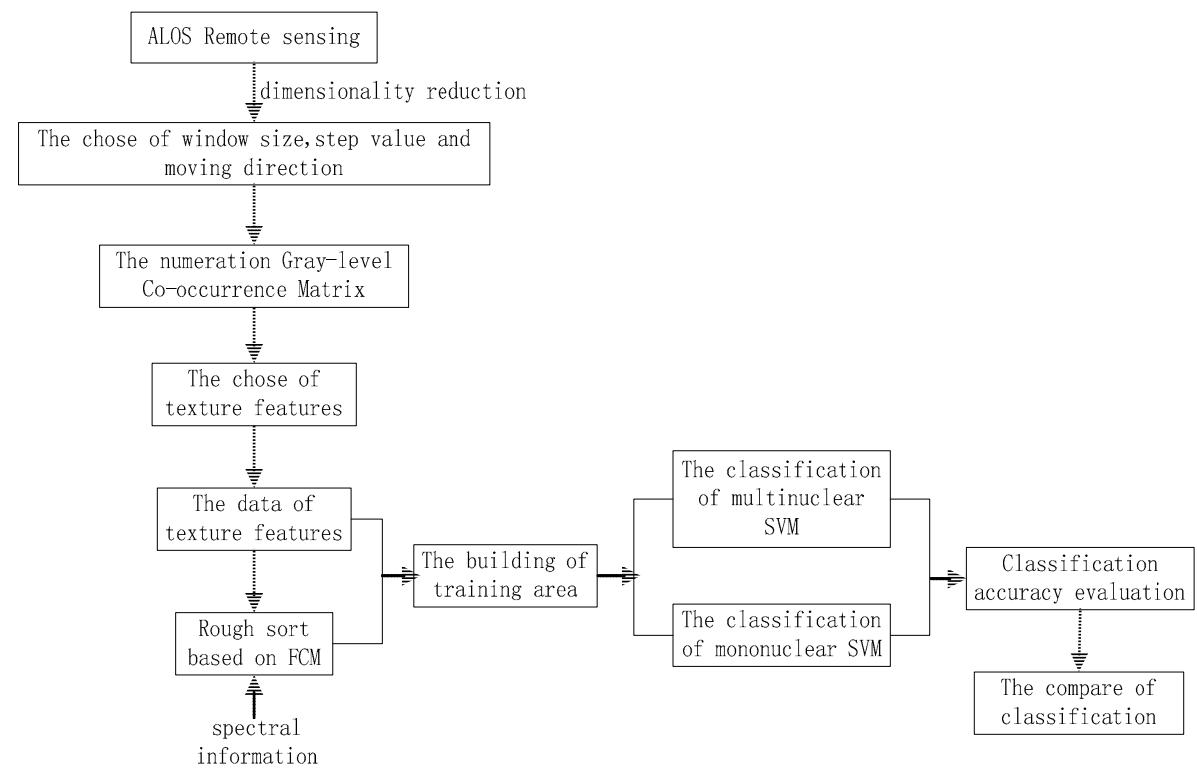

Fig. 1. This experiment process flow chart

\section{Remote sensing image classification algorithm of coarse classification and textural features based on FCM}

For the supervised classification algorithm of the remote sensing image, the acquisition of the training samples is a very important step. Generally, the training samples are extracted with a method of combining the manual investigation and remote sensing image, but it takes more time and energy. Based on such defect, this paper proposes a method of carrying out coarse classification on the remote sensing image with FCM. This method includes selecting the training samples and reclassifying with a combination kernel function by combing the textural features of the image. Thus, the classification accuracy is improved well.

Basic principle of FCM. FCM clustering algorithm is the most famous fuzzy clustering analysis algorithm proposed by Bezdek in 1981 [7]. It is applied most widely and successfully among a large number of fuzzy clustering. In the following, the algorithm and its steps will be described by combining the remote sensing image.

Set the pixel count of the image to be subjected to the clustering analysis is $\mathbf{n}$. The image pixel set is $\mathrm{X}=\left\{x_{1}, x_{2}, \ldots, x_{n}\right\}$, wherein $\mathrm{x}_{k}=\left\{x_{k}^{1}, x_{k}^{2} \ldots, x_{k}^{p}\right\}^{T}$, and $\mathrm{p}$ is the number of band. The image pixel is divided into c categories. The clustering center of each category is $\mathrm{v}_{t}=\left\{v_{t}^{1}, v_{t}^{2} \ldots, v_{t}^{p}\right\}^{T}$, and $u_{t k}$ is used to express that the pixel $x_{k}$ belongs to the membership degree which takes $i$ as the center. The membership matrix $u$ is defined as follows:

$$
\mathrm{U}=\left[u_{t k}\right]_{e \times n} .
$$

The matrix U shall meet the following constraint conditions: 


$$
\left\{\begin{array}{l}
\sum_{\mathrm{k}=1}^{\mathrm{n}} \mathrm{u}_{\mathrm{lk}}>0 \\
\sum_{\mathrm{l}=1}^{\mathrm{c}} \mathrm{u}_{\mathrm{lk}}=1, \\
0 \leq \mathrm{u}_{\mathrm{lk}} \leq 1(\mathrm{i}=1,2, \ldots, \mathrm{c} ; \mathrm{k}=1,2, \ldots, \mathrm{n}) .
\end{array}\right.
$$

The membership degree $u_{1 k}$ is fuzzified, and the value range of $u_{1 k}$ is the number from 0 to 1 . In this way, a pixel can also belong to different categories.

In the algorithm proposed by Qirui Huang, et al. in the literature [6], the objective function J is defined as:

$$
\mathrm{Im}_{\mathrm{m}}(\mathrm{U}, \mathrm{V})=\Sigma_{\mathrm{k}=1}^{\mathrm{n}} \sum_{\mathrm{l}=1}^{\mathrm{c}}\left(\mathrm{u}_{\mathrm{lk}}\right)^{\mathrm{m}} \cdot\left(\mathrm{d}_{\mathrm{lk}}\right)^{2}
$$

$\left(\mathrm{d}_{\mathrm{k}}\right)^{2}=\left\|\mathrm{x}_{\mathrm{k}}-\mathrm{v}_{1}\right\|^{2}$ is Euclidean distance. $\mathrm{m} \in[1, \infty]$ is a fuzzy weighted exponent.

In traditional unsupervised classification algorithm, spectral characteristics of each category change over time, terrain and the like. Spectrum cluster groups among different images cannot maintain its continuity, and hard to contrast. Now the general classification of remote sensing image uses the method of supervised classification. However, the subjective factor is strong in supervised classification algorithms. In addition, selection and assessment of training samples need to spend more time and manpower, which is an important reason for the development of SVM in remote sensing image. For this defect, Yunhong Ju, et al. proposed the method that k-means extracts training samples in literature [8], which used k-means clustering again after each type of sample with k-means clustering algorithm. But when extracting training samples, the method was relatively low. The presence of mixed pixels made some pixels difficult to be accurately divided. FCM can overcome shortcomings of the hard division to some extent. Therefore, application of FCM in remote sensing image classification is wider. For the shortcomings of local optimum of FCM, the literature [9] proposed an improved FCM clustering algorithm based on the GK and GG, which had better adaptability for different shapes, sizes and densities of the clusters and the accuracy of the classification was improved.

After full consideration and based on mixed pixel problem of remote sensing data, this paper firstly carries out the coarse classification with FCM, and combines with texture features to participate in the classification.

Gray-level co-occurrence matrix. The co-occurrence matrix is a matrix constituted by joint probability density $[P(i, j, d, \theta)]$ among the image gray levels, which reflects the spatial correlation of the gray level between any two points in the image $[10]$. The GLCM $[P(i, j, d, \theta)]_{L \times L}$, wherein, $\theta$ is defined as direction; $\mathrm{d}$ is defined as the interval; $[P(i, j, d, \theta)]$ is the value of the element in the $i$-th row and the $j$-th line of the co-occurrence matrix, which is the probability of presenting the gray level $\mathrm{j}$ by taking the gray level $\mathrm{i}$ as the starting point when the spatial distance $\mathrm{d}$ and the direction $\theta$ are given. $\mathrm{L}$ is the number of the gray level; and $\theta$ is generally taken $0^{\circ}, 45^{\circ}, 90^{\circ}$ and $135^{\circ}$ [11].

As the GLCM cannot be directly used for describing the textural features of the image, people define some statistics to extract the textural features reflected by it. The common method mainly includes the following [12]:

1) Mean

$$
\sum_{\mathrm{l}=0}^{L-1} \sum_{\mathrm{l}=0}^{\mathrm{L}-1} \mathbf{i} \cdot \mathrm{p}(\mathrm{i}, \mathrm{j})
$$

Mean reflects the average situation of gray.

2) Variance

$$
\sum_{\mathrm{l}=0}^{\mathrm{L}-1} \sum_{\mathrm{l}=0}^{\mathrm{L}-1}(\mathrm{i}-\mathrm{u})^{2} \cdot \mathrm{p}(\mathrm{i}, \mathrm{j})
$$


$\mathrm{u}$ is the mean of $\mathrm{P}(\mathrm{i}, \mathrm{j})$, and variance reflects the change in the size of the gray.

3) Inverse gap

$$
\sum_{1=0}^{L-1} \sum_{\mathrm{l}=0}^{L-1} \frac{\mathrm{p}(1,1)}{1+(1-1)^{2}}
$$

The inverse gap reflects the local homogeneity. When the co-occurrence matrix concentrates along the diagonal, the inverse gap is larger.

4) Contrast

$$
\sum_{\mathrm{l}=0}^{\mathrm{L}-1} \mathrm{n}^{2}\left\{\sum_{\mathrm{l}=0}^{\mathrm{L}-1} \sum_{\mathrm{l}=0}^{\mathrm{L}-1} \mathrm{p}(\mathrm{i}, \mathrm{j})\right\} \text {. }
$$

The contrast reflects the impact of the clarity of texture. The texture grooves deeper, the greater the contrast, the better the image of visual clarity.

5) Angular two moment (energy)

$$
\sum_{\mathrm{l}=0}^{\mathrm{L}-1} \sum_{\mathrm{l}=0}^{\mathrm{L}-1} \mathrm{p}(\mathrm{i}, \mathrm{j}) \mathrm{j}^{2} .
$$

Angular two moment reflects the gray distribution uniformity of the image .Energy moment of coarse texture is large. Energy moment of fine texture is small.

6) Correlation

$$
\sum_{\mathrm{l}=0}^{\mathrm{L}-1} \sum_{\mathrm{l}=0}^{\mathrm{L}-1} \frac{1 \mathrm{1} p(1,1)-\mathrm{u}_{1} \mathrm{u}_{2}}{\sigma_{1}^{2} \sigma_{2}^{2}} .
$$

$\mu_{1}, \mu_{2}, \sigma_{1}, \sigma_{2}$ is variance and mean.

According to the characteristics of remote sensing images selected in the paper, the four selected statistical indicators can be better highlighted image textures. In general, there are four moving directions can be chosen, i.e. $0^{\circ}, 45^{\circ}, 90^{\circ}, 135^{\circ}$. The step between the center pixel and immediately adjacent pixel generally is 1-pixel, 3-pixels and so on.1-pixel, 3-pixels and 5-pixels are selected in this paper. Finally, this test proves that 1-pixel is the best.

SVM. SVM is a pattern recognition method based on statistical theory, mainly used in pattern recognition. It is often difficult to divide low-dimensional space vector set. Thus, the solution is to map them to the high-dimensional space which increases the complexity of the calculation, but the kernel function is just to solve this problem.

Each kernel function has its own advantages and disadvantages, and different kernel functions exhibit different characteristics; the role of different kernel functions constructed SVM is also different. Kernel function currently used to make SVM divide into two categories: global and local kernel functions. Global kernel functions have global characteristics, in which distant data points can have an impact on the value of the kernel function; local kernel functions have locality which allow closed data points have an impact on the value of the kernel functions.

The RBF kernel function and the Sigmoid kernel function are used in this paper. The Sigmoid kernel function is a global kernel function, coming from neural network. However, the RBF kernel function is a local kernel function. When combining the two experiments above, this test can obtain not only the local information but also the global information of the test points.

\section{Analysis on classification result}

In the test, ALOS remote sensing image in Yinchuan Yuehai Park is used as the test data. The 
size of $10 \mathrm{~m}$ multispectral remote sensing image is $512 \times 512$. Based on the visual result and actually investigated result, this paper selects four ground object samples: water, plants, residents and sand. The original image is shown in Fig.2:

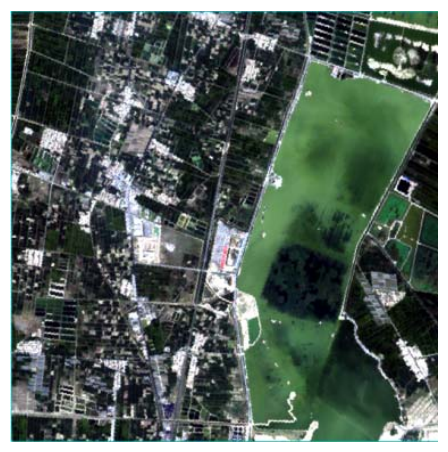

Fig. 2. Original ALOS image

In consideration of the problems of calculation amount and runtime, the original image is firstly subjected to the dimensionality reduction with the principal component analysis (PCA) in the test. Because the proportion of characteristic value of the first principal component accounts for $85 \%$, the first principal component of the image is selected so that the textural features of the second stage of the classification in this paper are extracted. With the method, the coarse classification effects of FCM and k-means are contrasted in the first stage. The maximum iterations of the FCM are 200, and the default values are adopted by other parameters. In the Fig.3, it indicates the result of FCM classification and the result of k-means classification. Since the k-means is the unsupervised classification, some pixels cannot be accurately classified due to the existence of mixed pixels. As can be seen from Fig.3, a small part of plants in Fig.3 (b) is divided into water. The more sand is also divided into the residents. Such situation is improved by the classification result of Fig.3 (a).

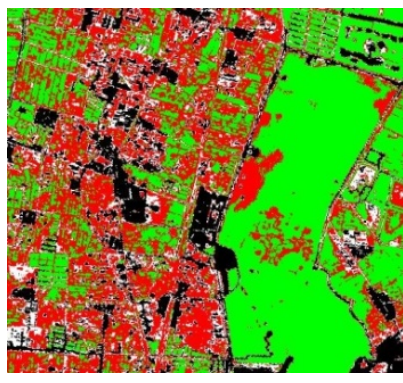

Water
Plants

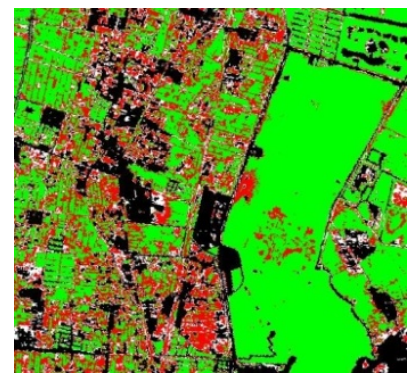

Sand
Residents
(a) Classification result of FCM
(b) Classification result $\mathrm{K}$ means

Fig. 3. Classification result of FCM and k-means

In order to use the information of the original image better, this paper carries out the textural feature extraction on the image with the GLCM. By means of the research objectives, calculation amount and extraction algorithms, four textural features i.e. correlation, contrast, energy and uniformity are finally selected; four directions, i.e. $0^{\circ}, 45^{\circ}, 90^{\circ}$ and $135^{\circ}$, are selected. Finally, the four textural features are obtained by seeking the average values and variances of the four directions. After the observation and contrast, the correlation is finally selected for participating in the classification. In the end, the test is carried out by selecting the final parameters of which the window is $5 \times 5$ and the moving step length is 1 . The four textures characteristic diagram is shown in Fig.4. 


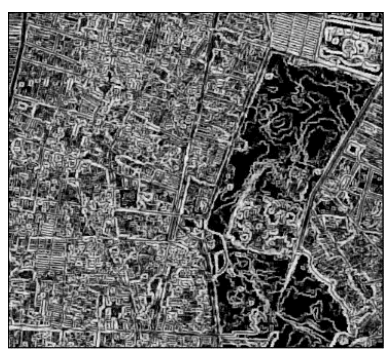

(a) Correlation

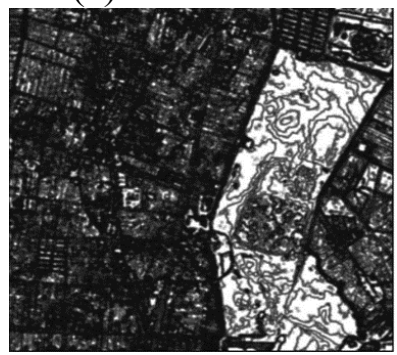

(c) Energy

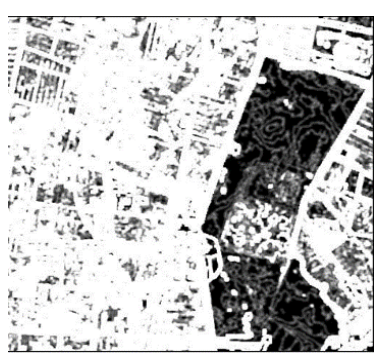

(b) Contrast

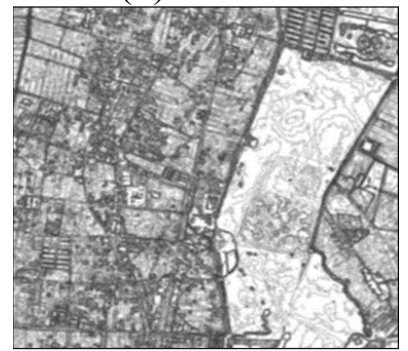

(d) Homogeneity

Fig. 4. Texture feature comparison chart

In order to achieve the better classification effect, this paper randomly selects the classification result to participate in the classification of the SVM on the basis of the classification of FCM. On the basis of observing the data characteristics and testing repeatedly, the classification parameters which are comparatively suitable for the wet land are selected and the comparison is showed on the Fig.5. When the maximum likelihood classification algorithm in the supervised classification is used for classifying, result in the Fig. 5 shows that the classification effects of the water and plants are bad, many wrong classifications are presented. The degree of fragmentation is relatively big. For the classification result of SVM, the kernel function adopts the RBF kernel function. It can be seen from the result figure that the degree of fragmentation is also very big, but is superior to the maximum likelihood classification result. In the Fig. 6, the wrong classification phenomenon is presented in the residents and water. A part of water and plants has the crossover and wrong classification phenomena. By contrasting the original image and carrying out field investigation, the classification effect of the method in this paper is relatively good; the border, profile and some other details can be reflected well; the classification effect of the water and plants is relatively good; but there is still the wrong classification and a certain degree of fragmentation showing on the residents and water. The comparison diagram of the final classification result is shown in Fig.5.

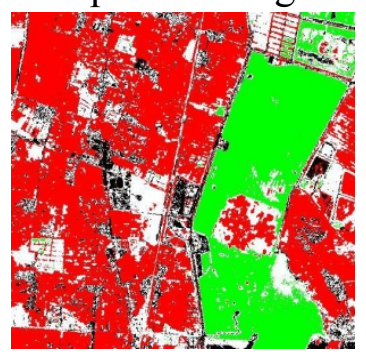

Water

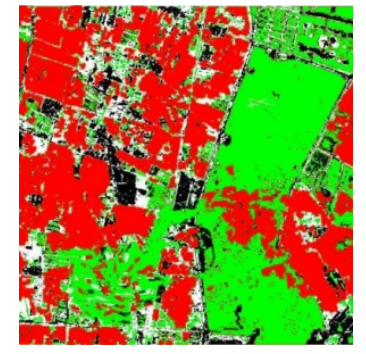

Sand

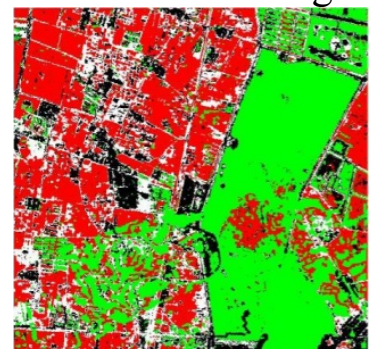

Residents

Fig. 5. Results of classification. (a) Maximum likelihood classification.

(b) SVM classification. (c) The method classification

In order to quantitatively compare the classification result, the accuracy evaluation shall be carried out on the result after the remote sensing image classification. This paper carries out the quantitative evaluation on the classification result with the confusion matrix. The evaluation result of the classification accuracy shall be shown in Table 1. As can be seen from the accuracy analysis table of the classification result, compared with the maximum likelihood classification and the SVM, the overall classification accuracy is improved to some extent. Especially when the combination kernel function is used, kappa's coefficient is improved from 0.6898 to 0.8743 . 
Table 1. Result evaluation of classification accuracy

\begin{tabular}{c|c|c}
\hline Algorithm & Classification Accuracy/\% & Kappa Coefficient \\
\hline $\begin{array}{c}\text { Maximum likelihood } \\
\text { classification }\end{array}$ & 79.085 & 0.6898 \\
\hline SVM classification & 86.182 & 0.8058 \\
\hline The method classification & 91.273 & 0.8743 \\
\hline
\end{tabular}

In order to verify the validity and feasibility of the method, another local image in Yuehai Park is classified with the method in this paper. Landsat data is used. The original data is shown in Fig.6. Four ground object samples, i.e. water, plants, residents and sand are selected according to the visual result and actually investigated result. The classification result of the image is shown in Fig.7.

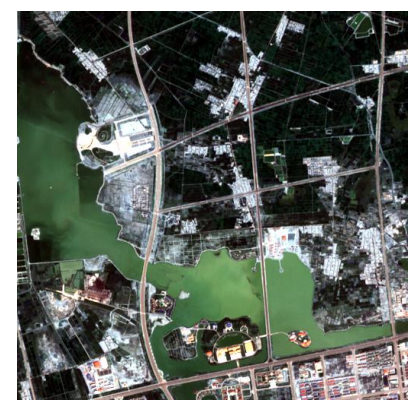

Fig. 6. Original ALOS image

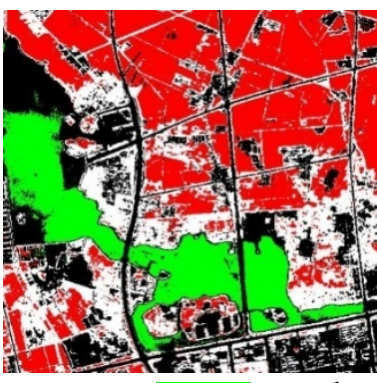

Water

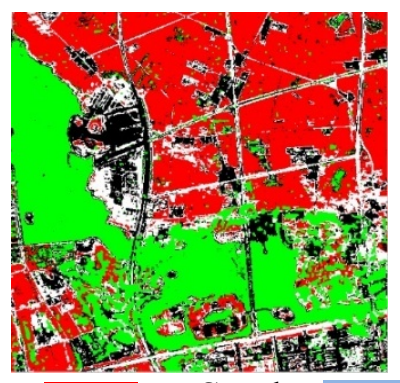

Sand

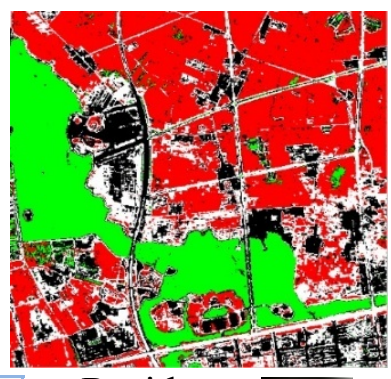

Residents

Fig. 7. Result of classification. (a) Maximum likelihood classification.

(b) SVM classification. (c) The method classification

Compared to the other two classification algorithm, it can be seen from Fig. 7 that the error of maximum likelihood classification algorithm is greater, i.e. misclassification of water and residents is more serious; there is cross misclassification phenomenon among some water and plants. The result of SVM classification can reduce the phenomenon of misclassification. But it has a greater degree of fragmentation. Through field visits and comparing to the original image, the proposed classification method of the paper is better, i.e. it reduces the degree of fragmentation; classification of water and plants is better; some of the boundaries and contours are clear.

Similarly, in order to compare the effect of classification of quantitative, the confusion matrix is used for accuracy assessment. The result of evaluation of classification accuracy is shown in Table 2. Comparing the two results, it can be seen that the method of FCM classification which selects training samples with texture features is better than maximum likelihood classification and SVM. The overall classification accuracy is improved.

Table 2. Result evaluation of classification accuracy

\begin{tabular}{c|c|c}
\hline Algorithm & Classification Accuracy/\% & Kappa Coefficient \\
\hline $\begin{array}{c}\text { Maximum likelihood } \\
\text { classification }\end{array}$ & 80.607 & 0.7219 \\
\hline SVM classification & 84.363 & 0.7775 \\
\hline The method classification & 92.364 & 0.8898 \\
\hline
\end{tabular}

The training sample shall be manually selected for the traditional algorithm based on SVM. The method used in this paper is a method which is free of manual intervention and randomly select the 
training sample through FCM and make the screened textural features participate in the classification. After the comparative study and analysis of the above instances, it indicates that the method in this paper is a classification method which is more effective than the traditional method.

\section{Conclusion}

In view of the disadvantages of the traditional remote sensing image classification method, this paper firstly carries out the coarse classification on it with FCM. Four textural features of the image are extracted with GLCM. The better textural feature is screened from them and used as the classification feature vector to participate in the classification. The classification model is built according to the advantages of SVM on solving the small sample, sub-linearity and others. The test shows that the method in this paper improves the accuracy significantly by comparing with the traditional classification method. However, it can be seen from the result figure that the wrong classification phenomenon is still presented by the residents and water. The best parameter can be found by multiple tests in the future study, or the combination of the kernel function is changed so as to be more beneficial to the further improvement of the classification accuracy.

\section{Acknowledgments}

This work is supported by National Natural Science Foundation of China(Project No. 611 62013,61461003 ) and the graduate innovation project of 2014 Beifang University for Nationa lities.

\section{References}

[1] LIU L F, CHEN Y H, LIJ. Texture analysis methods used in remote sensing images [J]. Remote Sensing Technology and Application, 2003, 18(6):441-447(in Chinese).

[2] VAPNIK V N. An overview of statistical learning theory [J]. IEEE Transactions on Neural Networks, 1999, 10(5): 988-999.

[3] Chen Liang, Youjing Zhang, Chen Bo. High Spatial Resolution Remote Sensing Image Classification based on Decision Tree Classification Combined with Multi-scale Texture [J]. Geography and Geo-Information Science, 2007, 23(4): 18-21.

[4] Qian Yin, Ping Guo. Multispectral Remote Sensing Image Classification with Multiple Features [J]. IEEE, 2007, 3(12): 360-365.

[5] Lu Liu, Taiyong Wang, Algorithm of Texture Segmentation Combining FCM and FSVM [J]. IEEE, 2009, 4(23): 294-297.

[6] Qirui Huang, Guangmin Wu, Jianming Chen, Hequn Chu. Automated Remote Sensing Image Classification Method Based on FCM and SVM [J]. IEEE, 2012, 4(23): 1-4.

[7] Liew A, Leung S H, LauW H, et al.. Segmentation of ColorLip Images by Spatial Fuzzy Clustering [J]. IEEE Trans. on Fuzzy Systems, 11 (4): 542-549, 2003.

[8] HongyunJu, Zhang Junben, Li Chaofeng etc. Remote sensing image based on K-means and SVM automatic classification method [J]. Application Research of computers, 2007, 24(11): 318-320.

[9] YU J, GUO P, CHEN P, et al. Remote sensing image classification based on inprover fuzzy C-means [J]. Geo-spatial Information Science, 2008, 11(2):90-94.

[10] Srinivasa G N, Shobha G. statistical texture analysis. Proc. of World Academy of Science, Enginecring and Technology, 2008, 36:1264-1270.

[11] Ma Li, Fan Yingyue. Image texture analysis, 2009.

[12] Haralick R M, Shanmugam, Dinstein I H. Textural features for image classification. IEEE Trans.on SMC, 2000, 3(6):610-621. 